OPEN ACCESS

Edited by:

Markus J. Tamás,

University of Gothenburg, Sweden

Reviewed by:

Per Hägglund,

Technical University of Denmark,

Denmark

Jonas Warringer,

University of Gothenburg, Sweden

*Correspondence:

Raymond J. Turner

turnerr@ucalgary.ca

Specialty section:

This article was submitted to

Cellular Biochemistry,

a section of the journal

Frontiers in Molecular Biosciences

Received: 30 September 2015 Accepted: 29 November 2015

Published: 18 December 2015

Citation:

Vrionis HA, Wang S, Haslam B and Turner RJ (2015) Selenite Protection of Tellurite Toxicity Toward Escherichia

coli. Front. Mol. Biosci. 2:69.

doi: 10.3389/fmolb.2015.00069

\section{Selenite Protection of Tellurite Toxicity Toward Escherichia coli}

\author{
Helen A. Vrionis, Siyuan Wang, Bronwyn Haslam and Raymond J. Turner* \\ Department of Biological Sciences, University of Calgary, Calgary, AB, Canada
}

In this work the influence of selenite on metal resistance in Escherichia coli was examined. Both synergistic and antagonistic resistance and toxicities were found upon co exposure with selenite. In wild type cells co-exposure to selenite had little effect on arsenic resistance, decreased resistance to cadmium and mercury but led to a dramatically increased resistance to tellurite of 32-fold. Due to the potential importance of thiol chemistry in metal biochemistry, deletion strains in $\gamma$-glutamylcysteine synthetase (key step in glutathione biosynthesis, encoded by $g s h A)$, thioredoxin $(\operatorname{tr} x A)$, glutaredoxin $(g r x A)$, glutathione oxidoreductase ( $g \circ r)$, and the periplasmic glutathione transporter $(c y d D)$ were also evaluated for resistance to various metals in the presence of selenite. The protective effect of selenite on tellurite toxicity was seen in several of the mutants and was pronounced in the gshA mutant were resistance to tellurite was increased up to 1000 -fold relative to growth in the absence of selenite. Thiol oxidation studies revealed a faster rate of loss of reduced thiol content in the cell with selenite than with tellurite, indicating differential thiol reactivity. Selenite addition resulted in reactive oxygen species (ROS) production equivalent to levels associated with $\mathrm{H}_{2} \mathrm{O}_{2}$ addition. Tellurite addition resulted in considerably lower ROS generation while vanadate and chromate treatment did not increase ROS production above that of background. This work shows increased resistance toward most oxyanions in mutants of thiol redox suggesting that metalloid reaction with thiol components such as glutathione actually enhances toxicity of some metalloids.

Keywords: metalloids, glutathione, tellurite, selenite, metal resistance

\section{INTRODUCTION}

The increasing use of tellurite in industrial applications has resulted in release into the environment creating a health concern (Ding et al., 2002; Ba et al., 2010; Sandoval et al., 2010) and an interest in better understanding the molecular mechanism of tellurite toxicity. Toxicity of tellurite in many enteric bacteria occurs at concentrations as low as $1 \mu \mathrm{g} / \mathrm{mL}$ (Turner et al., 1999; Bajaj and Winter, 2014). A number of plasmid and chromosomally encoded determinants for tellurite resistance have been identified (Jobling and Ritchie, 1987; Walter and Taylor, 1989; Hill et al., 1993; Taylor et al., 1994; Turner et al., 1995a,b; Taylor, 1997) and though the mechanisms of resistance have not been fully elucidated in many of these, evidence has shown that the mechanism of resistance is not through increased efflux or reduced uptake of tellurite (Turner et al., 1995a).

Thiol redox enzymes (glutathione reductase and thioredoxin reductase) and their metabolites (thioredoxin, glutaredoxin, and glutathione) which are all part of the cellular thiol:redox buffering system have been shown to be involved in oxyanion chemistry (Turner et al., 1995b). Glutathione 
has been shown to be a key intermediate in cellular processing of selenium and is likely a primary target of tellurite reactivity (Turner et al., 2001). When species of microbes are exposed to tellurite one can observe a blackening of the media, which is the result of reduction of tellurite to elemental tellurium $\left(\mathrm{Te}^{0}\right)$, which can accumulate as nanocrystals or nanoparticles (Turner et al., 2012). It has been proposed that glutathione is capable of mediating tellurite to elemental tellurium $\left(\mathrm{Te}^{0}\right)$ reduction (Turner et al., 2001), and this reduction can be observed in vitro. The reaction may also be accompanied with generation of superoxide anions as has been seen with selenite (Bebien et al., 2002; Kessi and Hanselmann, 2004).

In response to environmental stresses, microbes can exhibit a variety of protective mechanisms. Pre-exposure to a contaminant at sub lethal levels can lead to increased resistance to subsequent exposure of the same stressor (adaptive response) or exposure to a different stress (cross-protective response; Vattanaviboon et al., 2003). Such protective effects have been observed to contribute to adaptation to oxidative stress and metal toxicity (Banjerdkij et al., 2005). In Xanthomonas campestris for instance, cadmium exposure has been shown to provide cells with protection against peroxide killing (Banjerdkij et al., 2005). In the present work, coexposure of Escherichia coli strains to selenite is seen to increase resistance to a number of metals including tellurite. Being in the same group in the periodic table does not guarantee equivalent ROS production and this was observed in this work where the ROS production by tellurite was considerably lower than that of selenite. This was in line with previous observations (Tremaroli et al., 2007) suggesting that the protective effect provided by selenite may be related to it's triggering a stronger adaptation to oxidative stress.

\section{MATERIALS AND METHODS}

\section{Strains and Growth Conditions}

Strains utilized in this work was derived from the Keio collection (Datsenko and Wanner, 2000; Baba et al., 2006). Strain K12 (W3110) BW25113 (F (araD-araB)567_lacZ4787(::rrnB-3)_rph1 _(rhaD-rhaB) 568 hsdR514 was used as Wild type. Subsequent mutants contain a Kanamycin insertion into the designated gene: JW2663 ( $\Delta g s h A)$, JW3467 ( $\Delta$ gor), JW0833 ( $\Delta$ grxA), JW5856 $(\triangle \operatorname{trx} A)$, and JW0870 $(\triangle c y d D)$. Cultures were streaked from frozen stocks onto LB media with $40 \mu \mathrm{g} / \mathrm{ml} \mathrm{Kan}$ and grown at $37^{\circ} \mathrm{C}$ for $24 \mathrm{~h}$-these first streaks (maintenance cultures-MC) were stored for approximately 2 weeks at $4^{\circ} \mathrm{C}$. As needed, fresh second streaks or liquid cultures were made from these cultures and grown for $24 \mathrm{~h}$ (over-night) prior to experimentation. Under the culture conditions used in this work there was no significant difference in growth rate observed for the mutants relative to WT.

\section{Stock Solutions}

Metal salts were obtained from Sigma Chemical Company (St. Louis, U.S.A.) for the various metal(loids): selenite $\left(\mathrm{Na}_{2} \mathrm{SeO}_{3}\right)$, tellurite $\left(\mathrm{Na}_{2} \mathrm{TeO}_{3}\right)$, vanadate $\left(\mathrm{NaVO}_{3}\right)$, chromate $\left(\mathrm{Na}_{2} \mathrm{CrO}_{4}\right)$, arsenite $\left(\mathrm{NaAsO}_{2}\right)$, cadmium $\left(\mathrm{CdCl}_{2}\right)$, and mercury $\left(\mathrm{HgCl}_{2}\right)$ were diluted in sterile water at twice the highest concentration to be tested (Turner et al., 2001). Metal solutions were passed through a $0.22 \mu \mathrm{m}$ syringe into sterile vials and stored at room temperature.

\section{Minimal Inhibitory Concentration (MIC)}

Minimal inhibitory concentration was assessed in 96 well microtitre plates. One column served as sterile control and one as a no metal control. Ten columns were used for susceptibility testing for the $\log$-2 dilution series of the metal(loid). Two rows for each metal were used for technical replicates within a plate. Each experiment was performed three times thus providing 6 replicates for each metal concentration. Metal concentration ranges tested were $4096-8 \mu \mathrm{g} / \mathrm{ml}$ arsenite, $410-0.4 \mu \mathrm{g} / \mathrm{ml}$ chromate, $3200-31.3 \mu \mathrm{g} / \mathrm{ml}$ vanadate, $40000-$ $390 \mu \mathrm{g} / \mathrm{ml}$ selenite, and $512-0.025 \mu \mathrm{g} / \mathrm{ml}$ tellurite. A working solution of metal was prepared (highest concentration to be tested) in Luria Bertaini broth media and subsequent concentrations achieved by serial dilution. Inoculums were prepared as previously described (Harrison et al., 2005). Briefly, a second sub-culture was prepared from MC $24 \mathrm{~h}$ prior to susceptibility testing experiments and used to create a standard matching a 1.0 McFarland Standard. This solution was diluted $1 / 30$ in $\mathrm{LB}$ and $10 \mu \mathrm{L}$ of this dilution was added to each well of the microtitre plate. Total volume in each well was $210 \mu \mathrm{l}(200 \mu \mathrm{l}$ $\mathrm{LB}$ and $10 \mu \mathrm{l}$ inoculum).

An adaptation of the interaction index defined by Berenbaum can be defined by $\Sigma$ FIC $=$ FIC $_{\mathrm{A}}+\mathrm{FIC}_{\mathrm{B}}=\mathrm{MIC}_{\mathrm{A}} / \mathrm{MIC}_{\mathrm{AB}}+$ $\mathrm{MIC}_{\mathrm{B}} / \mathrm{MIC}_{\mathrm{BA}}$; where $\mathrm{MIC}_{\mathrm{A}}, \mathrm{MIC}_{\mathrm{B}}$ are the MICs of metals $\mathrm{A}$ and $\mathrm{B}$ acting alone and $\mathrm{MIC}_{\mathrm{AB}}, \mathrm{MIC}_{\mathrm{BA}}$ are the MICs of metals acting in combination. $\Sigma F_{\text {FIC }}$ is the lowest $\Sigma$ FIC when synergy is occuring or the highest, $\Sigma \mathrm{FIC}_{\max }$, for antagonism (Berenbaum, 1977; Bellio et al., 2015). In this approach an FIC value of less than 0.5 indicates synergy while values above 4 indicate antagonism.

\section{Thiol Assays}

Over-night cultures were diluted 1/100 in M9 media and grown at $37^{\circ} \mathrm{C}(250 \mathrm{rpm})$ to mid-log phase $\left(\mathrm{O}^{-\mathrm{D}_{0.600}} \sim 0.5\right)$. This bulk culture was then divided into four $25 \mathrm{~mL}$ aliquots in sterile flasks containing a stir-bar and moved to a stir plate placed at room temperature. The four test conditions settled on for this work were as follows: control (no metal added); tellurite $(1 \mu \mathrm{g} / \mathrm{mL})$; selenite $(800 \mu \mathrm{g} / \mathrm{mL})$ and tellurite + selenite $(1 \mu \mathrm{g} / \mathrm{mL}$ and $800 \mu \mathrm{g} / \mathrm{mL}$ respectively). After an $\sim 5 \mathrm{~min}$ room temperature equilibration, time zero samples were collected for determination of initial protein and thiol content. $1 \mathrm{~mL}$ samples were collected and pelleted by centrifugation and the supernatant discarded. Samples were then immediately frozen in liquid $\mathrm{N}_{2}$ while the pellets for protein determination were washed once with water prior to flash-freezing. Samples were stored at $-80^{\circ} \mathrm{C}$ until analysis. Subsequent samples were taken over the $2 \mathrm{~h}$ exposure. Samples for protein concentration determination were taken at the 0,1 , and $2 \mathrm{~h}$ time points.

Thiol determination was by the dithiol-nitrobenzoate (DTNB) assay (Turner et al., 1999). In brief, a solution containing $0.1 \mathrm{~mm}$ DTNB, $5 \mathrm{mM}$ EDTA, $50 \mathrm{mM}$ Tris $\mathrm{pH}=8$ and $0.1 \%$ SDS was prepared. The lysis was performed in the presence of DTNB so that reaction with DTNB would be rapid. It is possible that some thiol oxidation would occur during the cell lysis but 
this is expected to be constant across all samples. Each sample pellet after thawing on ice was resuspended by vortexing with $1 \mathrm{~mL}$ of DTNB solution and incubated at $37^{\circ} \mathrm{C}$ for $45 \mathrm{~min}$. Samples were pelleted by centrifugation and the Absorbance of the supernatants at $412 \mathrm{~nm}$ was measured. An extinction coefficient for DTNB of $1.36 \times 10^{4} \mathrm{M}^{-1} \mathrm{~cm}^{-1}$ was used to determine reduced thiol concentration. Protein concentration was determined using the Lowry method (Lowry et al., 1951) with bovine serum albumin (BSA) used to develop a standard curve.

\section{Reactive Oxygen Species (ROS) Assay}

An overnight culture was diluted $1 / 100$ in fresh LB and grown at $37^{\circ} \mathrm{C}(250 \mathrm{rpm})$ to mid-log phase $\left(\mathrm{O}^{\mathrm{D}} \mathrm{D}_{0.600} \sim 0.5\right)$. Cells were pelleted, washed one time in $0.9 \%$ saline and then resuspended in an equivalent volume of $0.9 \%$ saline. A small aliquot was collected and pelleted for protein determination. DCFA was added to the cell/saline solution from a $2 \mathrm{mM}$ Stock to a final concentration of $5 \mu \mathrm{M}$. DCFA is light sensitive so from here on all solution flasks/vials were kept covered. The cell/saline/dye solution (sample) was shaken for $1 \mathrm{~h}$ at room temperature. The sample solution was then distributed in $4 \mathrm{~mL}$ aliquots into cuvettes and appropriate volumes of metals were added from metal stock solutions to obtain desired final concentrations $(128 \mu \mathrm{g} / \mathrm{ml}$ $\mathrm{AsO}_{2}^{-}, 512 \mu \mathrm{g} / \mathrm{ml} \mathrm{AsO}_{2}^{-}, 13 \mu \mathrm{g} / \mathrm{ml} \mathrm{Cr}_{2} \mathrm{O}_{7}, 16384 \mu \mathrm{g} / \mathrm{ml} \mathrm{Se}_{2} \mathrm{O}_{4}$, $800 \mu \mathrm{g} / \mathrm{ml} \mathrm{Se}_{2} \mathrm{O}_{3}, 0.125,0.25,1$, and $25 \mu \mathrm{g} / \mathrm{ml} \mathrm{TeO}{ }_{3}, 800 \mu \mathrm{g} / \mathrm{ml}$ $\mathrm{VO}_{3}$ ). Cell/metal mixes were quickly mixed by inversion and time marked as $t=0$. No metal and $1 \% \mathrm{H}_{2} \mathrm{O}_{2}$ addition were used as negative and positive controls respectively.

\section{Protein Isolation}

Over-night cultures were diluted $1 / 100$ in multiple $3 \mathrm{~L}$ flasks containing $1 \mathrm{~L}$ of fresh $\mathrm{LB}$ and culture grown to an O. $D_{0.600} \sim 0.45-0.5$. Cells were harvested by centrifugation at $6000 \mathrm{rpm}$ for $10 \mathrm{~min}$. at $4^{\circ} \mathrm{C}$. Cells were then washed with $25 \mathrm{mM} \mathrm{K} \mathrm{HPO}_{4} / 75 \mathrm{mM} \mathrm{NaCl}(\mathrm{pH}=7)$ followed by another centrifugation. Cell pellets were thoroughly resuspended in $25 \mathrm{mM} \mathrm{K} \mathrm{HPO}_{4}$ with $2 \mathrm{mM}$ Phenyl-methyl-sulfonyl fluoride (PMSF) and $0.03 \mathrm{mg} / \mathrm{mL}$ of DNAse I added. Cell suspensions (on ice) were then French pressed two times at 20,000 psi and subsequently centrifuged $(10,000 \mathrm{rpm}$ for $15 \mathrm{~min}$ ) to remove unlysed cells and debris. Soluble and membrane fractions were separated by ultracentrifugation at $40,000 \mathrm{rpm}$ for $1.5 \mathrm{~h}$ at $4^{\circ} \mathrm{C}$. Samples were stored at $-80^{\circ} \mathrm{C}$ until use.

\section{Superoxide Dismutase (SOD) Assay}

Soluble cell fractions were obtained as described above with the following modifications. Pellets (from $100 \mathrm{~mL}$ of cells) were washed once in phosphate buffered saline (PBS) and subsequently resuspended in $2 \mathrm{~mL}$ of $50 \mathrm{mM}$ Tris $(\mathrm{pH}=7.8)$ treated with $100 \mu \mathrm{M}$ PMSF. The cell suspensions were sonicated (on ice) on setting $50 \%$ power for two 1 min bursts with a $1 \mathrm{~min}$ pause in between and a final $10 \mathrm{~s}$ pulse. The suspensions were centrifuged at $7000 \mathrm{rpm} @ 4^{\circ} \mathrm{C}$ for $15 \mathrm{~min}$ and the supernatants (crude extracts) subsequently ultracentrifuged at $35200 \mathrm{rpm}$ for $1 \mathrm{~h}$. The supernatants (soluble fractions) were collected and stored at $-20^{\circ} \mathrm{C}$.
Approximately $35 \mu \mathrm{g}$ of soluble fraction for each of the samples were electrophoresed in non-denaturing $8 \%$ polyacrylamide gels at $4{ }^{\circ} \mathrm{C}$ and gel assay determination of SOD activity was performed by an in situ staining procedure as previously described (Beauchamp and Fridovich, 1971; Dhindsa et al., 1981; Borsetti et al., 2005). In brief, gels were equilibrated in $50 \mathrm{mM}$ potassium phosphate buffer and then treated with a mixture of nitrotetrazolium blue, riboflavin, and TEMED in the dark. The solution was then poured away and gel(s) exposed to light. A dark band indicates SOD activity. Bands corresponding to SOD activity were analyzed by an Image Analyzer FLA-3000 (Fujifilm, Japan).

\section{RESULTS}

\section{Susceptibility to Metals and Co-exposure}

The susceptibility of $E$. coli wild type and mutants of thiol/redox homeostasis $(\triangle g s h A, \Delta g r x A, \Delta \operatorname{tr} x A, \Delta g o r$, and $\Delta c y d D)$ toward $\mathrm{NaAsO}_{2}, \mathrm{CdCl}_{2}, \mathrm{HgCl}_{2}, \mathrm{Na}_{2} \mathrm{TeO}_{3}$, and $\mathrm{Na}_{2} \mathrm{SeO}_{3}$ was assayed to determine their MIC. The nature of the two-log dilution, differences in MIC at the low concentration range are less meaningful than changes at higher concentrations. All strains exhibited a high inherent resistance to $\mathrm{NaAsO}_{2}$ and $\mathrm{CdCl}_{2}$ (256-1024 $\mu \mathrm{g} / \mathrm{mL}$ and $64-256 \mu \mathrm{g} / \mathrm{mL}$ respectively), while being less resistant to $\mathrm{HgCl}_{2}(\sim 2 \mu \mathrm{g} / \mathrm{mL})$ and $\mathrm{TeO}_{3}^{2-}(\sim 0.25 \mu \mathrm{g} / \mathrm{mL})$ (Table 1).

As expected from previous studies exploring tellurite, the MIC thiol redox mutants were less than wildtype (Turner et al., 1995b). The deletion of gor exhibited a slightly higher susceptibility to $\mathrm{CdCl}_{2}$ relative to the other strains. The $\triangle \operatorname{tr} x A$ mutant showed a loss of resistance to $\mathrm{AsO}_{2}^{-}$. The $\Delta c y d D$ mutant led to changes in MIC for $\mathrm{AsO}_{2}^{-}$, Cd as well as $\mathrm{TeO}_{3}^{2-}$.

The effect of cell co-exposure to the above metals with increasing (2-fold) concentrations of $\mathrm{SeO}_{3}^{2-}(0-3200 \mu \mathrm{g} / \mathrm{mL})$ was also examined (Table 1). The maximum of $3200 \mu \mathrm{g} / \mathrm{ml}$ was chosen, as this is the dilution before the MIC of Selenite. The goal was to see if selenite would protect cells from the mechanisms of other metal toxicities. This combined with the key thiol/redox homeostasis mutants would provide clues to biochemical processes. We considered that both synergistic and antagonistic results could be possible. Arsenite resistance was not affected by the presence of $\mathrm{SeO}_{3}^{2-}$ until the selenite MIC was approached $(\sim 3200 \mu \mathrm{g} / \mathrm{mL})$. $\mathrm{CdCl}_{2}$ resistance decreased very slowly at lower selenite concentrations $(\sim 0-200 \mu \mathrm{g} / \mathrm{mL})$ but dropped to $<8 \mu \mathrm{g} / \mathrm{ml}$ when more than $400 \mu \mathrm{g} / \mathrm{ml}$ of $\mathrm{SeO}_{3}^{2-}$ was present in the growth media. Cell resistance to $\mathrm{HgCl}_{2}$ did not change considerably with selenite co-exposure, while in contrast selenite provided remarkable protection against tellurite toxicity in all the strains, with the gsh $A$ mutant exhibiting up to 1000 fold higher MIC than wild type. It should be noted that the protective effect was specific to selenite (i.e., selenate did not provide protection, data not shown). The synergy occurring during co-treatment with tellurite and selenite was revealed by an interactive model where FIC values were below 0.5 indicating synergy; while FIC values for all other metals were above 4 reflecting the antagonism with these metals. 
TABLE 1 | Evaluation of synergistic and antagonistic responses of the addition of selenite on the minimal inhibitory concentration (MIC) toward other metal(loid)s.

\begin{tabular}{|c|c|c|c|c|c|c|c|c|}
\hline \multirow[b]{3}{*}{ Strain } & \multirow[b]{3}{*}{ Metal(loid) } & \multicolumn{7}{|c|}{ Selenite addition $\mu \mathrm{g} / \mathrm{mL}$} \\
\hline & & 0 & 100 & 200 & 400 & 800 & 1600 & 3200 \\
\hline & & \multicolumn{7}{|c|}{ MIC $(\mu \mathrm{g} / \mathrm{mL})$} \\
\hline \multirow[t]{4}{*}{ WT } & $\mathrm{AsO}_{2}^{-}$ & 512 & 512 & 512 & 512 & 512 & 256 & $<8$ \\
\hline & $\mathrm{Cd}^{2+}$ & 256 & 128 & 16 & $<8$ & $<8$ & $<8$ & $<8$ \\
\hline & $\mathrm{Hg}^{2+}$ & 2 & 1 & 1 & 1 & $<0.5$ & $<0.5$ & $<0.5$ \\
\hline & $\mathrm{TeO}_{3}^{2-}$ & 1 & 2 & 4 & 8 & 16 & 32 & 2 \\
\hline \multirow[t]{4}{*}{$\Delta \operatorname{trxA}$} & $\mathrm{AsO}_{2}^{-}$ & 256 & 256 & 256 & 128 & 128 & $<8$ & $<8$ \\
\hline & $\mathrm{Cd}^{2+}$ & 256 & 64 & 16 & $<8$ & $<8$ & $<8$ & $<4$ \\
\hline & $\mathrm{Hg}^{2+}$ & 2 & $<0.5$ & $<0.5$ & $<0.5$ & $<0.5$ & $<0.5$ & $<0.5$ \\
\hline & $\mathrm{TeO}_{3}^{2-}$ & 0.25 & 4 & 16 & 64 & 128 & 32 & 16 \\
\hline \multirow[t]{4}{*}{$\Delta g r x A$} & $\mathrm{AsO}_{2}^{-}$ & 512 & 256 & 256 & 128 & 128 & 16 & $<8$ \\
\hline & $\mathrm{Cd}^{2+}$ & 256 & 64 & 16 & $<8$ & $<8$ & $<8$ & $<8$ \\
\hline & $\mathrm{Hg}^{2+}$ & 2 & 2 & 1 & 1 & 0.5 & 1 & $<0.5$ \\
\hline & $\mathrm{TeO}_{3}^{2-}$ & 0.25 & 2 & 4 & 32 & 32 & 32 & $<1$ \\
\hline \multirow[t]{4}{*}{$\Delta \mathrm{gsh} A$} & $\mathrm{AsO}_{2}^{-}$ & 512 & 256 & 256 & 128 & 128 & 16 & $<8$ \\
\hline & $\mathrm{Cd}^{2+}$ & 128 & 64 & 32 & 32 & $<8$ & $<8$ & $<8$ \\
\hline & $\mathrm{Hg}^{2+}$ & 2 & 2 & $<0.5$ & $<0.5$ & $<0.5$ & $<0.5$ & $<0.5$ \\
\hline & $\mathrm{TeO}_{3}^{2-}$ & 0.25 & 64 & 128 & 256 & 256 & 64 & $<1$ \\
\hline \multirow[t]{4}{*}{$\Delta$ gor } & $\mathrm{AsO}_{2}^{-}$ & 512 & 512 & 512 & 256 & 32 & 8 & $<8$ \\
\hline & $\mathrm{Cd}^{2+}$ & 64 & 64 & 32 & 8 & $<8$ & $<8$ & $<8$ \\
\hline & $\mathrm{Hg}^{2+}$ & 2 & 1 & $<0.5$ & $<0.5$ & $<0.5$ & $<0.5$ & $<0.5$ \\
\hline & $\mathrm{TeO}_{3}^{2-}$ & 0.25 & 4 & 16 & 32 & 64 & 32 & $<1$ \\
\hline \multirow[t]{4}{*}{$\Delta c y d D$} & $\mathrm{AsO}_{2}^{-}$ & 1024 & 1024 & 1024 & 512 & 512 & 64 & $<8$ \\
\hline & $\mathrm{Cd}^{2+}$ & 128 & 64 & 32 & $<8$ & $<8$ & $<8$ & $<8$ \\
\hline & $\mathrm{Hg}^{2+}$ & 2 & 1 & 1 & $<0.5$ & $<0.5$ & $<0.5$ & $<0.5$ \\
\hline & $\mathrm{TeO}_{3}^{2-}$ & 0.5 & 8 & 16 & 32 & 64 & 64 & 64 \\
\hline
\end{tabular}

\section{Effect of $\mathrm{TeO}_{3}^{2-}, \mathrm{SeO}_{3}^{2-}$, and Co-exposure on RSH Content}

One of the targets of tellurite biochemistry is the reaction with free thiol groups mainly cysteine, which results in a depletion of cell RSH concentration (Turner et al., 2001). In order to examine if the mechanism of selenite protection is related to beneficial protection of the RSH pool, mid log phase cells were treated with either tellurite $(1 \mu \mathrm{g} / \mathrm{mL})$, selenite $(800 \mu \mathrm{g} / \mathrm{mL})$, or a mixture of the two and then changes in the RSH content over time monitored. Concentrations were chosen based on the MIC data in Table 1, where $800 \mu \mathrm{g} / \mathrm{mL}$ is the highest concentration with no inhibition found. Examination of the [RSH] with time curves in Figure 1 shows that in E. coli there is a rapid decrease in the RSH concentration in response to selenite addition. The response to tellurite is more gradual although the final RSH concentrations $2 \mathrm{~h}$ post-treatment are comparable. In the conditions when both $\mathrm{Se}$ and $\mathrm{Te}$ oxyanions are present, it is $\mathrm{SeO}_{3}^{2-}$ biochemistry that dominates over tellurite in driving RSH reactions. Greater decreases in RSH concentration in the $\Delta$ gor mutant reflect the decreased ability of this strain to reduce oxidized GSSG. For the $\triangle \operatorname{trx} A$ mutant $[\mathrm{RSH}]$ after $1 \mathrm{~h}$ was essentially identical in the tellurite and selenite ( \pm tellurite) conditions. A similar trend was seen in the $\Delta g r x A$ mutant. The $\Delta$ gor mutant exhibited the highest loss in $[\mathrm{RSH}]$ under all conditions including untreated. Furthermore, the $\Delta$ gor mutant exhibited the greatest difference in RSH content between the tellurite and selenite ( \pm tellurite) samples. The low detection of RSH levels and the minimal change in RSH under all conditions in the $\Delta g s h A$ mutant likely reflects the fact that the reactive (and DTNB detectable) [RSH] in E. coli is actually GSH.

\section{Production of Reactive Oxygen Species in Response to Oxyanion Exposure}

Mid-log phase cells were washed and incubated with a membrane permeable dye carboxy-dichlorodihydrofluorescein diacetate (DCFA) (Mishra et al., 2005) that fluoresces upon reaction with a variety of reactive oxygen species (ROS) as a result in cleavage of acetate and ester groups. Fluorescence from control (untreated) 


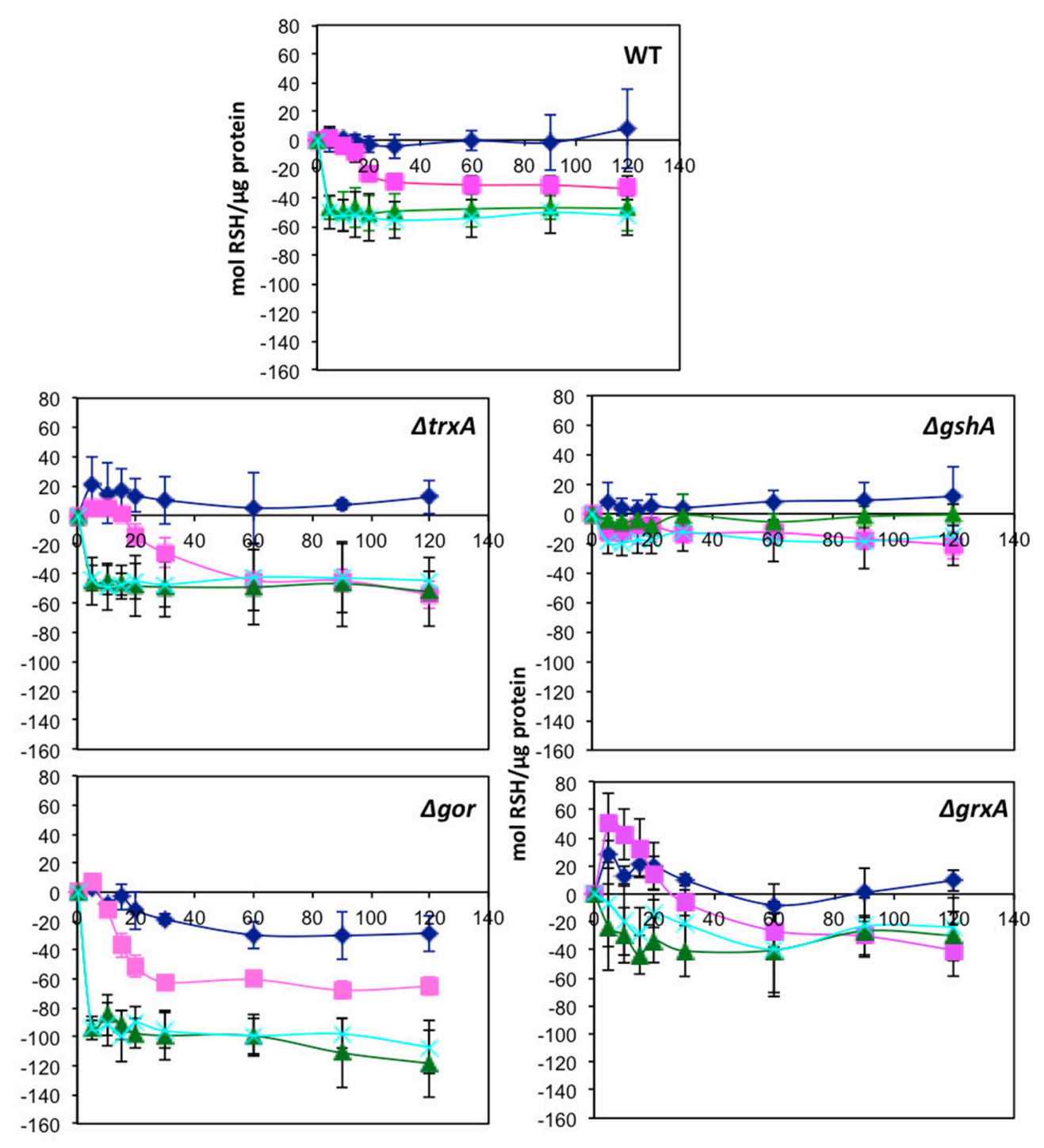

Time (min)

FIGURE 1 | Change in reduced thiol pool in response to various metalloid challenges. Mid log phase cultures were exposed to metalloid challenges and their reduced thiol content was measured with time to evaluate the thiol oxidation rate. Control without challenge (blue diamonds), $1 \mu \mathrm{g} / \mathrm{ml}$ tellurite (red squares), $800 \mu \mathrm{g} / \mathrm{ml}$ selenite (green triangles), $1 \mu \mathrm{g} / \mathrm{ml}$ tellurite with $800 \mu \mathrm{g} / \mathrm{ml}$ selenite (cyan X). Wild type is in upper panel with each of four different redox balance mutants indicated. The error bars represent the standard deviation between 3 biological trials.

cells was evaluated for $2 \mathrm{~h}$ post attack $[<8000$ arbitrary units (a.u.)] and background values were subtracted from subsequent fluorescence measurements.

The relative levels of ROS generated from selenite and tellurite and a series of other oxyanions (selenate, arsenite, chromate, and vanadate) were evaluated (Figure 2). Regardless of its high MIC, selenite exposure results in the highest ROS production exceeding that of the control hydrogen peroxide. Other oxyanions were added below their MIC levels. Arsenite and selenate provided similar ROS output even though the selenate was 32 -fold more concentrated. With arsenite used at one quarter the concentration resulted in a ROS response similar to that observed for $1 \mu \mathrm{g} / \mathrm{mL} \mathrm{TeO}_{3}^{2-}$. Neither $13 \mu \mathrm{g} / \mathrm{mL}$ chromate, nor $800 \mu \mathrm{g} / \mathrm{mL}$ vanadate were able to trigger a detectable ROS response in the cells under the conditions used.

Cells treated with selenite ( \pm tellurite, Figure 3 ) exhibited high fluorescence $(725,000$ and 800,000 a.u. respectively), comparable to cells treated with $1 \%$ hydrogen peroxide $(725,000$ a.u.). Tellurite treated cells exhibited a $\mathrm{TeO}_{3}^{2-}$ concentration dependent increased fluorescence reflecting increased ROS production with increased $\mathrm{TeO}_{3}^{2-}$ challenge. However, even at the highest oxyanion concentration $1 \mu \mathrm{g} / \mathrm{mL}$, the fluorescence was only $\sim 1 / 4$ of the fluorescence seen for the selenite treated cells. Cells treated with $25 \mu \mathrm{g} / \mathrm{mL} \mathrm{TeO}_{3}^{2-}$ (well above the MIC) 


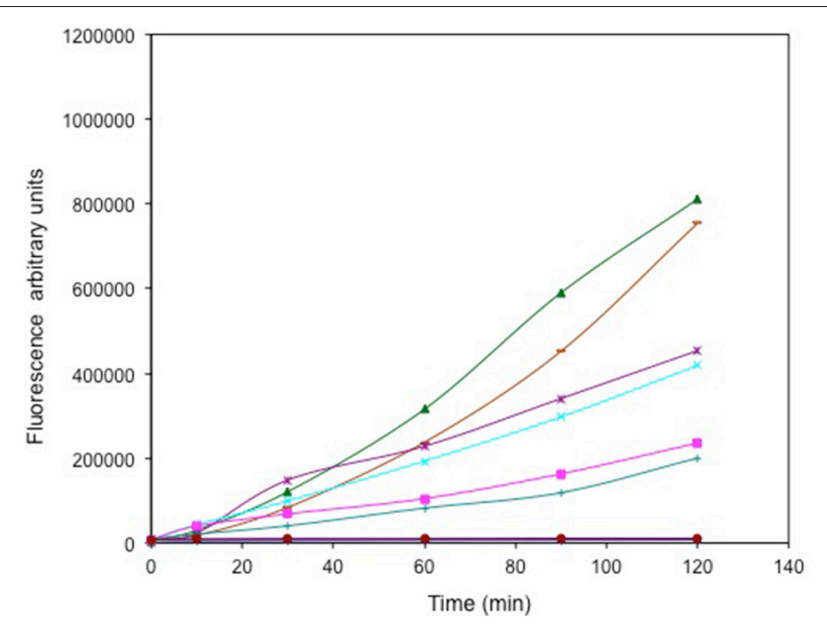

FIGURE 2 | Time course of reactive oxygen species (ROS) production. Exponentially growing cells were washed, suspended in saline in the presence of the ROS sensitive probe carboxy-DCFH-DA and exposed to metals. $800 \mu \mathrm{g} / \mathrm{mL}$ selenite (green triangle), $16384 \mu \mathrm{g} / \mathrm{mL}$ selenate (purple *), $512 \mu \mathrm{g} / \mathrm{mL}$ arsenite (cyan X), $1 \mu \mathrm{g} / \mathrm{mL}$ tellurite (pink square), $128 \mu \mathrm{g} / \mathrm{mL}$ arsenite (green +), $13 \mu \mathrm{g} / \mathrm{mL}$ chromate (blue line) and $800 \mu \mathrm{g} / \mathrm{mL}$ vanadate (brown circles). ROS production in non-treated (blue solid diamond) and cells treated with $\mathrm{H}_{2} \mathrm{O}_{2}$ (brown dash) are presented as negative and positive controls respectively. The error bars represent the standard deviation between 3 trials.

without selenite only showed a background level of fluorescence indicating that at this concentration the cells are killed and hence are not able to mount a ROS response. For the curve of addition of selenite at a level that increased the MIC of tellurite there was not much difference in ROS levels suggesting that ROS production is dominated by selenite.

\section{Effect of Oxyanions on Superoxide Dismutase (SOD) Activity}

Superoxide dismutase activity was evaluated using a zymogram in order to determine if selenite or tellurite inhibit or stimulate induction of this enzyme (Figure 4). Tellurite shows only an approximate doubling of the expression of Mn SOD (as evaluated by band intensities lanes 5 , untreated control, to lane 4 ). Selenite and selenite + tellurite were essentially equivalent leading to strong induction and activity of SOD. These results suggest that the selenite protective effect may partially be due to a differential dismutase enzyme accumulation/activity.

\section{Cell Viability Kill Curves Subsequent to Metal Exposure}

In order to evaluate the cell killing subsequent to metal exposure, the viability of log phase cells were examined for $2 \mathrm{~h}$ post metal addition by colony count plating. The shapes of these kill curves are different between the different conditions and thus are qualitatively compared. Although, the viability of cells treated only with tellurite dropped off rapidly at even $0.25 \mu \mathrm{g} / \mathrm{ml}$, co-exposure with selenite did not show any loss of viability even with $1 \mu \mathrm{g} / \mathrm{ml}$ tellurite (Figure 5A). This experiment was extended using different ratios of tellurite to selenite. High loads

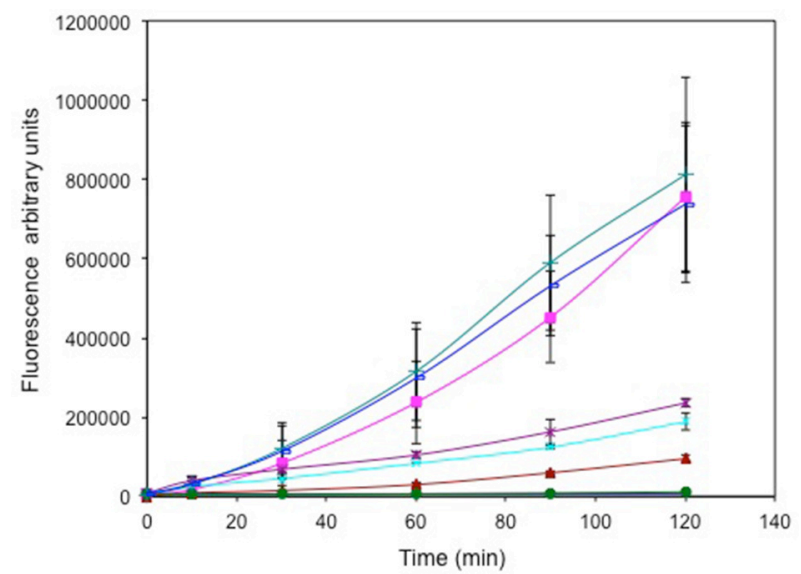

FIGURE 3 | Time course of reactive oxygen species (ROS) production. Exponentially growing cells were washed, suspended in saline in the presence of the ROS sensitive probe carboxy-DCFH-DA and exposed to metals. $800 \mu \mathrm{g} / \mathrm{mL}$ selenite (green triangle), $25 \mu \mathrm{g} / \mathrm{mL}$ tellurite/800 selenite (blue line), $1 \mu \mathrm{g} / \mathrm{mL}$ tellurite (brown *), $0.25 \mu \mathrm{g} / \mathrm{mL}$ tellurite (cyan X), $0.125 \mu \mathrm{g} / \mathrm{mL}$ tellurite (brown triangle), $25 \mu \mathrm{g} / \mathrm{mL}$ tellurite (solid green circle). ROS production in non-treated (blue solid diamond) and cells treated with $\mathrm{H}_{2} \mathrm{O}_{2}$ (pink square) are presented as negative and positive controls respectively. The error bars represent the standard deviation between 3 trials.

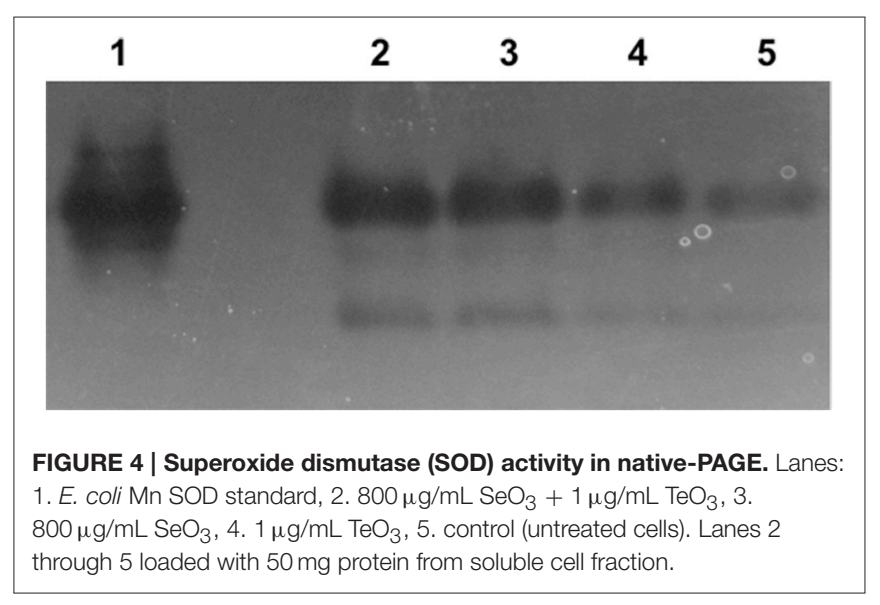

of selenite still provided protection of viability to extremely high loads of tellurite $(800 \mu \mathrm{g} / \mathrm{ml})$ (Figure 5B). The experiment was repeated using the $\triangle g s h A$ strain. In this strain there would be no glutathione as a target of either tellurite or selenite mediated Painter style reactions $\left(2 \mathrm{GSH}+\mathrm{Se}_{3}^{2-}->\mathrm{GS}-\mathrm{Se}-\right.$ SG) (Painter, 1941). We observe only minor loss of viability of tellurite-exposed cells, co -exposed with $800 \mu \mathrm{g} / \mathrm{mL}$ selenite, even at tellurite concentrations as extremely high as 1600 -fold over the MIC.

We note that in the $\triangle g s h A$ and $\Delta g o r$ strains grown in the presence of tellurite had markedly less blackening of the media from Te nanoparticle production (not shown), suggesting that in these strains reduction of tellurite was affected. A similar, though less dramatic, difference in the reduction to red elemental selenium was seen for the selenite treated cells. 


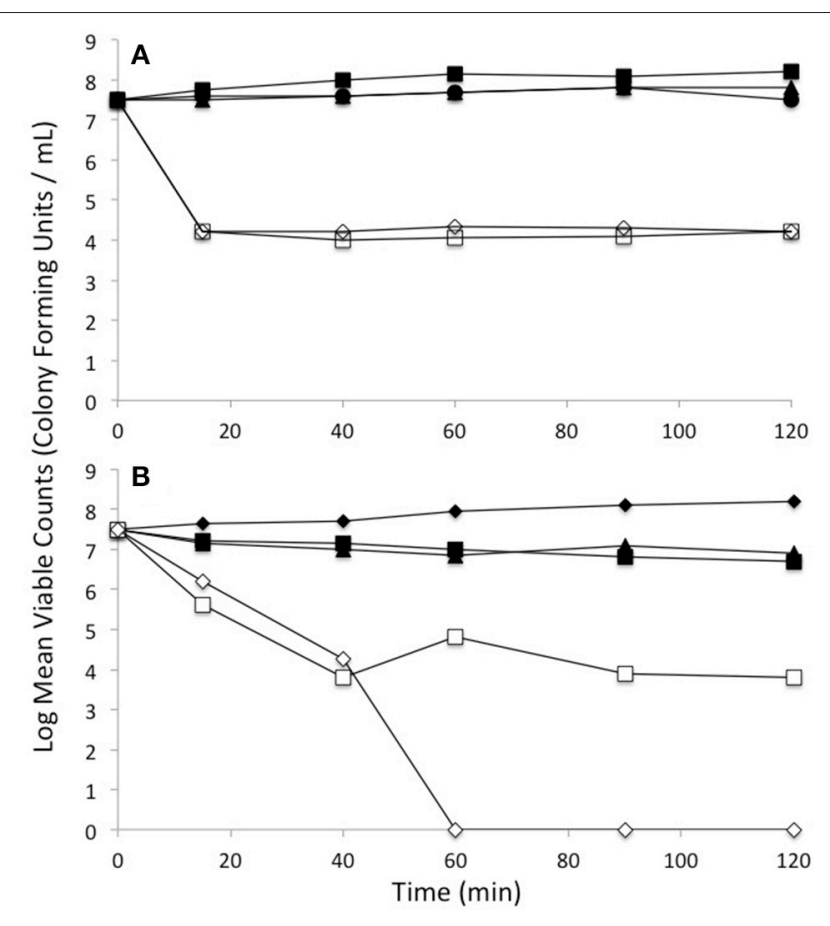

FIGURE 5 | Loss of viability upon exposure to selenite and tellurite combinations. (A) Wild type E. coli: Untreated (solid circle), $1 \mu \mathrm{g} / \mathrm{mL}$ tellurite (open dimond), $800 \mu \mathrm{g} / \mathrm{mL}$ selenite (solid square), $0.25 \mu \mathrm{g} / \mathrm{mL}$ tellurite (open square), $1 \mu \mathrm{g} / \mathrm{ml}$ tellurite with $800 \mu \mathrm{g} / \mathrm{ml}$ selenite (solid triangle). (B)

Comparison of selenite protection of tellurite toxicity between WT and a strain absent in glutathione production $\triangle$ gshA: WT strain no exposure (solid diamond), WT strain with $800 \mu \mathrm{g} / \mathrm{ml}$ tellurite and $800 \mu \mathrm{g} / \mathrm{ml}$ selenite (open square), WT strain with $1600 \mu \mathrm{g} / \mathrm{ml}$ tellurite and $800 \mu \mathrm{g} / \mathrm{ml}$ selenite (open triangle), $\Delta$ gshA strain with $800 \mu \mathrm{g} / \mathrm{ml}$ tellurite and $800 \mu \mathrm{g} / \mathrm{ml}$ selenite (solid triangle), $\Delta$ gshA strain with $1600 \mu \mathrm{g} / \mathrm{ml}$ tellurite and $800 \mu \mathrm{g} / \mathrm{ml}$ selenite (solid square). The standard deviations between experiments have magnitudes within the size of the plotting symbols.

\section{DISCUSSION}

Typically bacteria can reduce the toxicity of metals by decreasing their uptake, enhancing their efflux, by sequestration, metabolic by-pass, or through conversion (e.g., reduction/oxidation) to less toxic forms (Trutko et al., 2000; Lemire et al., 2013). For tellurite, reduction to elemental tellurium is evidenced by blackening of cells but the exact mechanism of tellurite reduction remains debated and is likely very different between bacterial species (Zannoni et al., 2008; Borghese et al., 2014; Zonaro et al., 2015). Some studies have proposed that tellurite reduction at the expense of glutathione and other thiols results in blocks in various biosynthetic processes and leads to cell death. Tellurite reduced inside the cell by un-related enzymatic activities, cytosolic glutathione and/or other reduced thiols generates metallic tellurium $\left(\mathrm{Te}^{0}\right)$ and superoxide leading many to propose that oxidative stress in response to metal exposure is one of the major causes of tellurite toxicity to cells (Zannoni et al., 2008; Chasteen et al., 2009). Reduction of selenite also involves reactions with sulfhydryl groups of thiol containing molecules such as glutathione (GSH) resulting in production of GS-Se-SG,
GS-she, and $\mathrm{HSe}^{-}$and finally elemental $\mathrm{Se}^{0}$ (Turner et al., 1998; Bebien et al., 2002). These reactions can produce damaging ROS molecules and expression of known oxidative stress defense mechanisms in bacterial cells has been shown to contribute to selenite resistance. A cross-protective effect of co-exposure to selenium and mercury has been documented in eukaryotes and has also been exhibited in the soil microbe Pseudomonas fluorescens (Belzile et al., 2006), and it is this possibility that we are exploring in this study for E. coli.

Some studies have described reductases exhibiting tellurite reducing ability (Moscoso et al., 1998) including nitrate reductases (Avazéri et al., 1997). In R. sphaeroides nitrate reductase reduction of tellurite has shown a low catalytic level and resistance could not be associated with the reduction (Sabaty et al., 2001). The nitrate reductases do not appear to be a factor in the current work, as the addition of nitrite or nitrate did not provide any change in MIC of tellurite toxicity and would not have been expected as the experiments here were performed aerobically. In E. coli 6phosphoguconate dehydrogenase (Sandoval et al., 2015) and NDH-II dehydrogenase (Díaz-Vásquez et al., 2014, 2015) appear to have tellurite reduction activity. In these studies, this reduction leads to superoxide production that affects aerobic electron transport chains leading to a move into an anaerobic respiratory state (Molina-Quiroz et al., 2014). Earlier work suggests that Glucose-6-phosphate dehydrogenase plays a protective role in the tellurite mediated oxidative stress (Sandoval et al., 2011). Overall the tellurite mediated stress on E. coli also affects the glycolytic pathway, changing the accumulation of various metabolic intermediates (Reinoso et al., 2012; Valdivia-González et al., 2012).

Numerous 'omic studies have been used to examine bacterial responses to metal toxicity (Bebien et al., 2002; Wang and Crowley, 2005; Brown et al., 2006; Chourey et al., 2008; Booth et al., 2011) and have shown that simultaneous induction of several stress response systems occurs. In E. coli, numerous enzymes with antioxidant properties are induced by selenite or selenate treatment including SodA and SodB (involved in degradation of the superoxide anion) and $\operatorname{Trx} A / \operatorname{Trx} B$ (involved in protection against $\mathrm{H}_{2} \mathrm{O}_{2}$ ) (Bebien et al., 2002). Examination of chromate stress in Shewanella oneidensis revealed a down-regulation of energy metabolism (electron transport components) and a similar up-regulation of functions associated with oxidative stress protection, protein stress protection and DNA repair. An increase in sulfur acquisition and assimilation elements at both the transcriptomic and proteomic level have also been observed (Brown et al., 2006). In Lactococcus lactis it has been demonstrated that tellurite induces different effects in two different Trx paralogs. A TrxA mutant exhibited decreased growth rate immediately following tellurite exposure whereas a TrxD mutant showed tellurite induced growth defects on a more long-term basis, indicating a potential role in detoxification (Björnberg et al., 2014; Efler et al., 2015). Here we see that selenite can protect the tellurite toxicity in a $\triangle \operatorname{tr} x A$ strain from a MIC of $1 \mu \mathrm{g} / \mathrm{mL}$ to a MIC of $128 \mu \mathrm{g} / \mathrm{ml}$ upon co-exposure with $800 \mu \mathrm{g} / \mathrm{mL}$ selenite. This suggests that the presence of this Thioredoxin may be behaving similar to 
that of glutathione, synergistically facilitating the toxicity of tellurite.

The structural similarity between chromate, selenite and tellurite and the biologically important anions $\mathrm{SO}_{4}^{2-}$ and $\mathrm{PO}_{4}^{3-}$ suggests that transport of $\mathrm{SeO}_{3}^{2-}$ and $\mathrm{TeO}_{3}^{2-}$ across the cell membrane likely occurs via the sulfate and phosphate transport systems. However, uptake has also been shown to be mediated by carboxylate transporters (Borghese et al., 2008). A transport blocking effect cannot however be the sole mechanism of selenium-protection, as all strains would then exhibit an equivalent level of protection.

An observation in this work was that ROS production does not correlate with MIC levels for different metals and increased ROS production does not necessarily correlate with increased metal toxicity. The addition of selenite resulted in higher ROS production (similar to that of hydrogen peroxide addition) than tellurite, although the latter is considerably more toxic to cells. This calls into question the belief that a large part of tellurite toxicity is attributable to its inducing an oxidative stress in cells. Cell death seems to occur at low concentrations of tellurite thus not providing enough oxyanion to catalyze high concentrations of ROS. The possibility exists that the low tellurite concentration of cell killing does not sufficiently trigger stress response mechanisms in the cell, limiting the cell's ability to mount an adaptive response to the metal. However, in a metabolomics investigation of a strain of Pseudomonas pseudoalcaligenes KF707, tellurite resistance correlated with the induction of oxidative stress response, resistance to membrane perturbation and reconfiguration of the cellular metabolism, particularly increased levels of glutathione (Tremaroli et al., 2009).

The protective effect of selenite may partially be attributed to its ability to trigger a greater oxidative stress and hence a stronger adaptive response but this is not the sole mechanism, as a similar protective effect would exist with other oxyanion combinations. Potential protection mechanisms include but are not limited to: triggering a stronger acute adaptive response (e.g., such as the stronger SOD response observed in this work) which is then protective against tellurite, preferential binding of selenite to tellurite target sites (these selenite products being less toxic), and alternate metabolic flux.

It is clear from this work that ROS associated cell damage is only part of the metal toxicity story. The ability of oxyanions to react with sulph-hydryl groups in protein cysteines and methionines as well as Fe-S clusters can result in disruption of function of a variety of proteins including components of the electron transport chain. The contribution of such interactions to tellurite toxicity is indicated by the increased sensitivity of cysteine desulfurase, iscS, mutants (Tantaleán et al., 2003) and other cysteine metabolism genes (e.g., cysK) in E. coli and other bacteria (O'Gara et al., 1997; Fuentes et al., 2007).

A number of roles for CydDC have been proposed and refuted including cysteine transport and haem transport (Cook and Poole, 2000). Strains mutated in $c y d D$ lack periplasmic cytochromes $c$ and do not assemble cytochrome $b_{562}$. CydD is not however needed for synthesis of haem $\mathrm{D}$ or assembly of CydA/CydB (encoding quinol oxidase cytochrome bd) (Cook and Poole, 2000). CydD has been shown to function as a GSH transporter and to be important for assembly of cytochrome $b d$ quinol oxidase (Pittman et al., 2005). This discovery indicates that GSH plays an important role in redox homeostasis in the periplasm and does not act alone in the cytoplasm. Cytochrome $b d$ has been shown to be induced when $E$. coli is grown under unfavorable growth conditions (Kato et al., 1996) and CydD mutants fail to synthesize periplasmic $c$-type cytochromes which are needed under anaerobic conditions or with alternate electron acceptors such as nitrite, as well as cytochrome $b d$ oxidase (Cook et al., 1997). The apparent lack of interference with tellurite reduction in a CydD mutant (indicated by blackening of growth media) disproves previous suggestions that cytochrome $b$ (or $d$ ) is involved in tellurite reduction. Yet we see increased tellurite toxicity protection by selenite in a $\Delta c y d D$ strain.

There have been studies into the role of the Dsb system proteins in cytochrome $c$ maturation, particularly through the $\mathrm{CcmG/H}$ pathways (Stirnimann et al., 2006). Pittman et al. (2005) in discovering that CydDC transports GSH to the periplasm, speculated that it mediated its activity by compensating for DsbD. The impaired ability of $\Delta g s h A$ and $\Delta g o r$ mutants to reduce tellurite supports a more direct effect of GSH on cytochrome proteins and reduction as has been previously suggested (Borsetti et al., 2007). Furthermore, the highly enhanced tellurite resistance with selenium protection in the $\triangle g s h A$ mutant suggests that the presence of GSH actually enhances tellurite toxicity and is similarly seen in $\Delta c y d D$ (Table 1). This is in agreement with the earlier work that suggested that the presence of $g s h A$ decreases survivability of $E$. coli by tellurite where as no other thiodisulphide metabolism gene showed a similar affect (Harrison et al., 2009).

The apparent slightly greater selenite effect on RSH reduction may largely be related to the 800 -fold higher addition of this metal relative to tellurite. The selenite driven reduction in RSH supports the observation of a greater oxidative stress with this metal. At the sensitivity tested, no additive effect of loss in RSH was observed in the double metal treatments. These results suggest that the increased toxicity of tellurite is not directly related to a greater reduction in the reduced thiol pool although it may be the nature of the products formed and not the concentration that results in toxicity.

This work presents the observation of a protective phenomenon rather than a synergistic destructive effect of the presence of the two metalloids selenite and tellurite. In addition this work shows that increased resistance observed by mutants of the various thiol redox pathway components suggests that metal reactions with thiol components such as GSH and associated oxygen radical production can actually enhance toxicity of certain metals. Alternatively, increased disulphide and oxidative stress associated with the disruption of the thiol redox buffering pathways may prime cell adaptive responses improving microbial survivability to metals.

\section{AUTHOR CONTRIBUTIONS}

HV was a PDF on the project leading the experiments, SW did technical work to support HV and followed up 
with key experimental repeats. $\mathrm{BH}$ was an undergraduate summer research student that did a lot of exploratory experiments. RT was the professor leading the project that advised and rethought experiments. HV and RT wrote the manuscript.

\section{REFERENCES}

Avazéri, C., Turner, R. J., Pommier, J., Weiner, J. H., Giordano, G., and Verméglio, A. (1997). Tellurite reductase activity of nitrate reductase is responsible for the basal resistance of Escherichia coli to tellurite. Microbiology 143, 1181-1189. doi: 10.1099/00221287-143-4-1181

Ba, L. A., Döring, M., Jamier, V., and Jacob, C. (2010). Tellurium: an element with great biological potency and potential. Org. Biomol. Chem. 8, 4203-4216. doi: $10.1039 / \mathrm{c} 0$ ob00086h

Baba, T., Ara, T., Hasegawa, M., Takai, Y., Okumura, Y., Baba, M., et al. (2006). Construction of Escherichia coli K-12 in-frame, single-gene knockout mutants: the Keio collection. Mol. Syst. Biol. 2, 2006-2008. doi: 10.1038/msb4100050

Bajaj, M., and Winter, J. (2014). Se (IV) triggers faster Te (IV) reduction by soil isolates of heterotrophic aerobic bacteria: formation of extracellular SeTe nanospheres. Microb. Cell Fact. 13, 168. doi: 10.1186/s12934-014-0168-2

Banjerdkij, P., Vattanaviboon, P., and Mongkolsuk, S. (2005). Exposure to cadmium elevates expression of genes in the OxyR and OhrR regulons and induces cross-resistance to peroxide killing treatment in Xanthomonas campestris. Appl. Environ. Microbiol. 71, 1843-1848. doi: 10.1128/AEM.71.4.1843-1849.2005

Beauchamp, C., and Fridovich, I. (1971). Superoxide dismutase: improved assays and an assay applicable to acrylamide gels. Anal. Biochem. 44, 276-287. doi: 10.1016/0003-2697(71)90370-8

Bebien, M., Lagniel, G., Garin, J., Touati, D., Verméglio, A., and Labarre, J. (2002). Involvement of superoxide dismutases in the response of Escherichia coli to selenium oxides. J. Bacteriol. 184, 1556-1564. doi: 10.1128/JB.184.6.15561564.2002

Bellio, P., Segatore, B., Mancini, A., Di Pietro, L., Bottoni, C., Sabatini, A., et al. (2015). Interaction between lichen secondary metabolites and antibiotics against clinical isolates methicillin-resistant Staphylococcus aureus strains. Phytomedicine 22, 223-230. doi: 10.1016/j.phymed.2014.12.005

Belzile, N., Wu, G. J., Chen, Y. W., and Appanna, V. D. (2006). Detoxificaiton of selenite and mercury by reduction and mutual protection in the assimilation of both elements by Pseudomonas fluorescens. Sci. Total Environ. 367, 704-714. doi: 10.1016/j.scitotenv.2006.03.008

Berenbaum, M. C. (1977). Synergy, additivism and antagonism in immunosuppression. A critical review. Clin. Exp. Immunol. 28, 1-18.

Björnberg, O., Efler, P., Ebong, E. D., Svensson, B., and Hägglund, P. (2014). Lactococcus lactis TrxD represents a subgroup of thioredoxins prevalent in Gram-positive bacteria containing WCXDC active site motifs. Arch. Biochem. Biophys. 15, 164-172. doi: 10.1016/j.abb.2014.09.010

Booth, S. C., Workentine, M. L., Weljie, A., and Turner, R. J. (2011). Metabolomics and its application to study metal toxicity. Metallomics 3, 1142-1152. doi: 10.1039/c1mt00070e

Borghese, R., Baccolini, C., Francia, F., Sabatino, P., Turner, R. J., and Zannoni, D. (2014). Reduction of chalcogen oxyanions and generation of nano-precipitates by the photosynthetic bacterium Rhodobacter capsulatus. J. Hazard. Mater. 269, 24-23. doi: 10.1016/j.jhazmat.2013.12.028

Borghese, R., Marchetti, D., and Zannoni, D. (2008). The highly toxic oxyanion tellurite ( $\mathrm{TeO} 3$-2) enters the phototrophic bacterium Rhodobacter capsulatus via an as yet uncharacterized monocarboxylate transport system. Arch. Microbiol. 189, 93-100. doi: 10.1007/s00203-007-0297-7

Borsetti, F., Francia, F., Turner, R. J., and Zannoni, D. (2007). The thiol:disulfide oxidoreductase DsbB mediates the oxidizing effects of the toxic metalloid tellurite (TeO32-) on the plasma membrane redox system of the facultative protroph Rhodobacter capsulatus. J. Bacteriol. 189, 851-859. doi: 10.1128/JB.01080-06

\section{ACKNOWLEDGMENTS}

The authors wish to thank Drs. V. Tremaroli, J. Harrison, D. Bay, and M. Workentine for input. This work was funded by an NSERC Discovery grant to RT.

Borsetti, F., Tremaroli, V., Michelacci, F., Borghese, R., Winterstein, C., Daldal, F., et al. (2005). Tellurite effects on Rhodobacter capsulatus cell viability and superoxide dismutase activity under oxidative stress conditions. Res. Microbiol. 156, 807-813. doi: 10.1016/j.resmic.2005.03.011

Brown, S. D., Thompson, M. R., Verberkmoes, N. C., Chourey, K., Shah, M., and Zhou, J. (2006). Molecular dynamics of the Shewanella oneidensis response to chromate stress. Mol. Cell. Proteomics 5, 1054-1071. doi: 10.1074/mcp.M500394-MCP200

Chasteen, T. G., Fuentes, D. E., Tantaleán, J. C., and Vásquez, C. C. (2009) Tellurite: history, oxidative stress and molecular mechanisms of resistance. FEMS Microbiol. Rev. 33, 820-832. doi: 10.1111/j.1574-6976.2009.00177.x

Chourey, K., Wei, W., Wan, X. F., and Thompson, D. K. (2008). Transcriptome analysis reveals response regulator SO2426-mediated gene expression in Shewanella oneidensis MR-1 under chromate challenge. BMC Genomics 9:395. doi: 10.1186/1471-2164-9-395

Cook, G. M., Membrillo-Hernández, J., and Poole, R. K. (1997). Transcriptional regulation of the cydDC operon, encoding a heterodimeric $\mathrm{ABC}$ transporter required for assembly of cytochromes $\mathrm{c}$ and bd in Escherichia coli K-12: regulation by oxygen and alternative electron acceptors. J. Bacteriol. 179, 6525-6530.

Cook, G. M., and Poole, R. K. (2000). Oxidase and periplasmic cytochrome assembly in Escherichia coli K-12: CydDC and CcmAB are not required for haem-membrane association. Microbiology 146, 527-536. doi: 10.1099/00221287-146-2-527

Datsenko, K. A., and Wanner, B. L. (2000). One-step inactivation of chromosomal genes in Escherichia coli K-12 using PCR products. Proc. Natl. Acad. Sci. U.S.A. 97, 6640-6645. doi: 10.1073/pnas.120163297

Dhindsa, R. S., Plumb-Dhindsa, P., and Thorpe, T. A. (1981). Leaf senescence: correlated with increased levels of membrane permeability and lipid peroxidation, and decreased levels of superoxide dismutase and catalase. J. Exp. Bot. 32, 93-101. doi: 10.1093/jxb/32.1.93

Díaz-Vásquez, W. A., Abarca-Lagunas, M. J., Arenas, F. A., Pinto, C. A., Cornejo, F. A., Wansapura, P. T., et al. (2014). Tellurite reduction by Escherichia coli NDH-II dehydrogenae results in superoxide production in membranes of toxicant-exposed cells. Biometals 27, 237-246. doi: 10.1007/s10534013-9701-8

Díaz-Vásquez, W. A., Abarca-Lagunas, M. J., Cornejo, F. A., Pinto, C. A., Arenas, F. A., and Vásquez, C. C. (2015). Tellurite-mediated damage to the Escherichia coli NDH-dehydrogenases and terminal oxidases in aerobic conditions. Arch. Biochem. Biophys. 566, 67-75. doi: 10.1016/j.abb.2014.10.011

Ding, W. J., Hasegawa, T., Peng, D., Hosaka, H., and Seko, Y. (2002). Preliminary investigation on the cytotoxicity of tellurite to cultured HeLa cells. J. Trace Elem. Med. Biol. 16, 99-102. doi: 10.1016/S0946-672X(02)80035-9

Efler, P., Kilstrup, M., Johnsen, S., Svensson, B., and Hägglund, P. (2015). Two Lactococcus lactis thioredoxin paralogues play different roles in responses to arsenate and oxidative stress. Microbiology 161(Pt 3), 528-538. doi: 10.1099/mic.0.000029

Fuentes, D. E., Fuentes, E. L., Castro, M. E., Pérez, J. M., Araya, M. A., Chasteen, T. G., et al. (2007). Cysteine metabolism-related genes and bacterial resistance to potassium tellurite. J. Bacteriol. 189, 8953-8960. doi: 10.1128/JB.01252-07

Harrison, J. J., Tremaroli, V., Stan, M. A., Chan, C. S., Vacchi-Suzzi, C., Heyne, B. J., et al. (2009). Chromosomal antioxidant genes have metal ion-specific roles as determinants of bacterial metal tolerance. Environ. Microbiol. 11, 2491-2509. doi: 10.1111/j.1462-2920.2009.01973.x

Harrison, J. J., Turner, R. J., and Ceri, H. (2005). Hight throughput metal susceptibility testing of microbial biofilms. BMC Microbiol. 5:53. doi: $10.1186 / 1471-2180-5-53$ 
Hill, S. M., Jobling, M. G., Lloyd, B. H., Strike, P., and Ritchie, D. A. (1993). Functional expression of the tellurite resistance determinant from the IncHI-2 plasmid pMER610. Mol. Gen. Genet. 241, 203-212. doi: 10.1007/BF00280218

Jobling, M. G., and Ritchie, D. A. (1987). Genetic and physical analysis of the plasmid genes expressing inducible resistance to tellurite in Escherichia coli. Mol. Gen. Genet. 208, 288-293. doi: 10.1007/BF00330455

Kato, C., Tamegai, H., Ikegami, A., Usami, R., and Horikoshi, K. (1996). Open reading frame 3 of the barotolerant bacterium strain DSS12 is complementary with cydD in Escherichia coli: cydD functions are required for cell stability at high pressure. J. Biochem. 120, 301-305. doi: 10.1093/oxfordjournals.jbchem.a021413

Kessi, J., and Hanselmann, K. W. (2004). Similarities between the abiotic reduction of selenite with glutathione and the dissimilatory reaction mediated by Rhodospirillum rubrum and Escherichia coli. J. Biol. Chem. 279, 50662-50669. doi: $10.1074 /$ jbc.M405887200

Lemire, J. A., Harrison, J. J., and Turner, R. J. (2013). Antimicrobial activity of metals: mechanisms, molecular targets and applications. Nat. Rev. Microbiol. 11, 371-384. doi: 10.1038/nrmicro3028

Lowry, O. H., Rosebrough, N. J., Farr, A. L., and Randall, R. J. (1951). Protein measurement with the Folin phenol reagent. J. Biol. Chem. 193, 265-275.

Mishra, B., Hassan, P. A., Priyadarsini, K. I., and Mohan, H. (2005). Reactions of biological oxidants with selenourea: formation of redox active nano selenium. J. Phys. Chem. B. 109, 12718-12723. doi: 10.1021/jp051328n

Molina-Quiroz, R. C., Loyola, D. E., Díaz-Vásquez, W. A., Arenas, F. A., Urzúa, U., Pérez-Donoso, J. M., et al. (2014). Global transcriptomic analysis uncovers a switch to anaerobic metabolism in tellurite-exposed Escherichia coli. Res. Microbiol. 165, 566-570. doi: 10.1016/j.resmic.2014.07.003

Moscoso, H., Saavedra, C., Loyola, C., Pichuantes, S., and Vasquez, C. (1998). Biochemical characterization of tellurite-reducing activities of Bacillus stearothermophilus V. Res. Microbiol. 149, 389-397. doi: 10.1016/S09232508(98)80321-5

O'Gara, J. P., Gomelsky, M., and Kaplan, S. (1997). Identification and molecular genetic analysis of multiple loci contributing to high-level tellurite resistance in Rhodobacter sphaeroides 2.4.1. Appl. Environ. Microbiol. 63, 4713-4720.

Painter, E. O. (1941). The chemistry and toxicity of selenium compounds with special reference to the selenium problem. Chem. Rev. 28, 179-213. doi: 10.1021/cr60090a001

Pittman, M. S., Robinson, H. C., and Poole, R. K. (2005). A bacterial glutathione transporter (Escherichia coli CydDC) exports reductant to the periplasm. J. Biol. Chem. 280, 32254-32261. doi: 10.1074/jbc.M503075200

Reinoso, C. A., Auger, C., Appanna, V. D., and Vásquez, C. C. (2012).Telluriteexposed Escherichia coli exhibits increased intracellular $\alpha$-ketoglutarate. Biochem. Biophys. Res. Commun. 421, 721-726. doi: 10.1016/j.bbrc.2012.04.069

Sabaty, M., Avazeri, C., Pignol, D., and Vermeglio, A. (2001).Characterization of the reduction of selenate and tellurite by nitrate reductases. Appl. Environ. Microbiol. 67, 5122-5126. doi: 10.1128/AEM.67.11.5122-5126.2001

Sandoval, J. M., Arenas, F. A., García, J. A., Díaz-Vásquez, W. A., ValdiviaGonzález, M., Sabotier, M., et al. (2015). Escherichia coli 6-phosphogluconate dehydrogenase aids in tellurite resistance by reducing the toxicant in a NADPH-dependent manner. Microbiol. Res. 177, 22-27. doi: 10.1016/j.micres.2015.05.002

Sandoval, J. M., Arenas, F. A., and Vásquez, C. C. (2011). Glucose-6-phosphate dehydrogenase protects Escherichia coli from tellurite-mediated oxidative stress. PLoS ONE 6:e25573. doi: 10.1371/journal.pone.0025573

Sandoval, J. M., Levêque, P., Gallez, B., Vasquez, C. C., and Buc Calderon, P. (2010). Tellurite-induced oxidative stress leads to cell death of murine hepatocarcinoma cells. Biometals 23, 623-632. doi: 10.1007/s10534-010-9316-2

Stirnimann, C. U., Grütter, M. G., Glockshuber, R., and Capitani, G. (2006). DsbD: a redox interaction hub in the Escherichia coli periplasm. Cell. Mol. Life Sci.. 63, 1642-1648. doi: 10.1007/s00018-006-6055-1

Tantaleán, J. C., Araya, M. A., Saavedra, C. P., Fuentes, D. E., Perez, J. M., Calderon, I. L., et al. (2003). The Geobacillus stearothermophilus V iscS gene, encoding cysteine desulfurase, confers resistance to potassium tellurite in Escherichia coli K-12. J. Bacteriol. 185, 5831-5837. doi: 10.1128/JB.185.19.5831-5837.2003

Taylor, D. E. (1997). Bacterial tellurite resistance. Trends Microbiol. 7, 111-115. doi: 10.1016/S0966-842X(99)01454-7
Taylor, D. E., Hou, Y., Turner, R. J., and Weiner, J. H. (1994). Location of a potassium tellurite resistance operon (tehAtehB) within the terminus of Escherichia coli K-12. J. Bacteriol. 176, 2740-2742.

Tremaroli, V., Fedi, S., and Zannoni, D. (2007). Evidence for a tellurite-dependent generation of reactive oxygen species and absence of a tellurite-mediated adaptive response to oxidative stress in cells of Pseudomonas pseudoalcaligenes KF707. Arch. Microbiol. 187, 127-135. doi: 10.1007/s00203-0060179-4

Tremaroli, V., Workentine, M. L., Weljie, A., Vogel, H., Ceri, H., Viti, C., et al. (2009). Metabolomics investigation of bacterial response to metal challenge. Appl. Environ. Microbiol. 75, 719-728. doi: 10.1128/AEM.01 771-08

Trutko, S. M., Akimenko, V. K., Suzina, N. E., Anisimova, L. A., Shlyapnikov, M. G., Baskunov, B. P., et al. (2000). Involvement of the respiratory chain of Gramnegative bacteria in the reduction of tellurite. Arch. Microbiol. 173, 178-186. doi: $10.1007 / \mathrm{s} 002039900123$

Turner, R. J., Aharonowitz, Y., Weiner, J. H., and Taylor, D. E. (2001). Glutathione is a target in tellurite toxicity and is protected by tellurite resistance determinants in Escherichia coli. Can. J. Microbiol. 47, 33-40. doi: 10.1139/cjm47-1-33

Turner, R. J., Borghese, R., and Zannoni, D. (2012). Microbial processing of tellurium as a tool in biotechnology. Biotechnol. Adv. 30, 954-963. doi: 10.1016/j.biotechadv.2011.08.018

Turner, R. J., Weiner, J. H., and Taylor, D. E. (1995a). Neither reduced uptake nor increased efflux is encoded by tellurite resistance determinants expressed in Escherichia coli. Can. J. Microbiol. 41, 92-98. doi: 10.1139/m95-012

Turner, R. J., Weiner, J. H., and Taylor, D. E. (1995b). The tellurite resistance determinants tehAtehB and klaAKlaBrelB have different biochemical requirements. Microbiology 141, 3133-3140. doi: 10.1099/13500872-141-123133

Turner, R. J., Weiner, J. H., and Taylor, D. E. (1998). Selenium metabolism in Escherichia coli. Biometals 11, 223-227. doi: 10.1023/A:1009290213301

Turner, R. J., Weiner, J. H., and Taylor, D. E. (1999). Tellurite mediated thiol oxidation in Escherichia coli. Microbiology 145, 2549-2557. doi: 10.1099/00221287-145-9-2549

Valdivia-González, M., Pérez-Donoso, J. M., and Vásquez, C. C. (2012). Effect of tellurite-mediated oxidative stress on the Escherichia coli glycolytic pathway. Biometals 25, 451-458. doi: 10.1007/s10534-012-9518-x

Vattanaviboon, P., Elamphungporn, W., and Mongkolsuk, S. (2003). Atypical adaptive and cross protective responses against peroxide killing in a bacterial plant pathogen Agrobacterium tumefaciens. Curr. Microbiol. 47, 323-326. doi: 10.1007/s00284-002-4011-3

Walter, E. G., and Taylor, D. E. (1989). Comparison of tellurite resistance determinants from IncP $\alpha$ plasmid RP4Ter and the IncHII plasmid pHH1508a. J. Bacteriol. 171, 2160-2165.

Wang, A., and Crowley, D. E. (2005). Global gene expression responses to cadmium toxicity in Escherichia coli. J. Bacteriol. 187, 3259-3266. doi: 10.1128/JB.187.9.3259-3266.2005

Zannoni, D., Borsetti, F., Harrison, J. J., and Turner, R. J. (2008). The bacterial response to the chalcogen metalloids Se and Te. Adv. Microb. Physiol. 53, 1-71. doi: 10.1016/S0065-2911(07)53001-8

Zonaro, E., Lampis, S., Qazi, S. J. S., Turner, R. J., and Vallini, G. (2015). Biogenic selenium and tellurium nanoparticles synthesized by environmental microbial isolates efficaciously inhibit bacterial planktonic cultures and biofilms. Front. Microbiol. 6:584. doi: 10.3389/fmicb.2015.00584

Conflict of Interest Statement: The authors declare that the research was conducted in the absence of any commercial or financial relationships that could be construed as a potential conflict of interest.

Copyright $(2015$ Vrionis, Wang, Haslam and Turner. This is an open-access article distributed under the terms of the Creative Commons Attribution License (CC BY). The use, distribution or reproduction in other forums is permitted, provided the original author(s) or licensor are credited and that the original publication in this journal is cited, in accordance with accepted academic practice. No use, distribution or reproduction is permitted which does not comply with these terms. 